\title{
Paragangliomas of the head and neck
}

\section{Reena George, MB BS, DMRD, MD \\ (Radiodiagnosis), MMed \\ Marie Grobbelaar, $\mathrm{MB} \mathrm{ChB}$ \\ Jaco du Plessis, MB ChB \\ Murray Hayes, $\mathbf{M B} \mathbf{C h B}$}

Department of Radiolology, Stellenbosch University, Tygerberg

\begin{abstract}
Paragangliomas of the head and neck are ubiquitous in their distribution, originating from the paraganglia or glomus cells within the carotid body, vagal nerve, middle ear, jugular foramen and numerous other locations. Imaging studies depict the location and extent of tumour involvement, help determine the surgical approach and help predict operative morbidity and mortality. The imaging features of common paragangliomas of the head and neck are illustrated in this article.
\end{abstract}

\section{Introduction}

The extra-adrenal neuro-endocrine system comprises an integrated and complex system of dispersed tissue throughout the body that possesses unique regulatory functions. A single collection of this tissue is called a paraganglion and the entire chain of tissue constitutes the paraganglia. They arise from neural crest progenitor cells and are therefore of neuro-ectodermal origin. Paragangliomas, the tumours of the paraganglia, arise from this specialised tissue at any site along these specific locations within the body.

Within the head and neck, the four most common sites are the carotid body at the common carotid artery (CCA) bifurcation, the jugular foramen, along the vagus nerve and within the middle ear. ${ }^{1}$ These masses produce characteristic findings on radiological images, particularly computed tomographic (CT), magnetic resonance (MR) imaging, and angiographic studies, as illustrated below. Less common sites include the sella turcica, pineal gland, cavernous sinus, larynx, orbit, thyroid gland, nasopharynx, mandible, soft palate, face, and cheek.

\section{Carotid body paraganglioma}

The typical CT appearance of a carotid body tumour is a well-defined soft-tissue mass within the carotid space of the infrahyoid neck. The underlying hypervascular- ity of the tumour results in homogeneous and intense enhancement following intravenous administration of contrast material. Splaying of the common carotid bifurcation is very suggestive of a carotid body tumour (Figs 1a and b). Rare manifestations include a heterogeneous pattern of enhancement due to focal thrombi or haemorrhage in larger lesions and superior extension
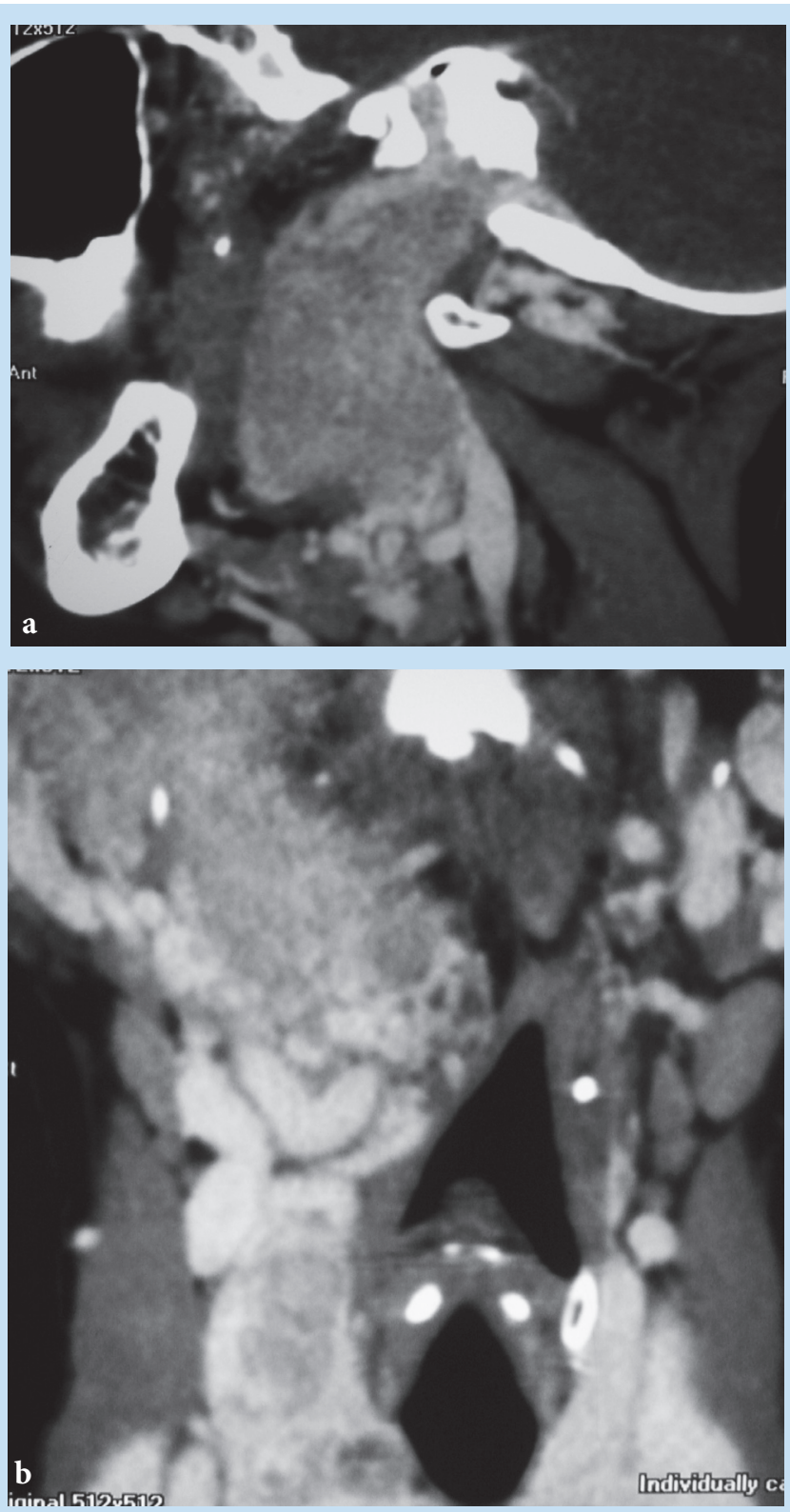

Fig. 1. Carotid body paraganglioma. (a) Sagittal reconstructions from a multidetector scan of the neck show an enhancing mass in the right side of the neck splaying the carotid bifurcation and extending to the skull base. (b) Coronal reconstructions of the same scan also clearly demonstrating the splaying of the carotid bifurcation by the mass. 


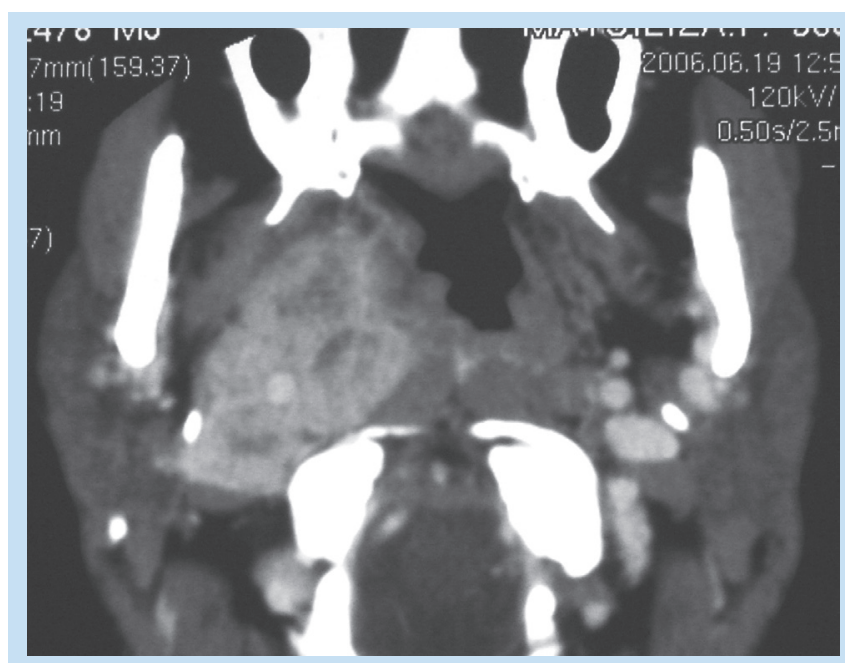

Fig. 2. Glomus vagale. Enhancing mass extending to the suprahyoid carotid space encasing the carotid vessels.

into the suprahyoid neck, seen in $8 \%$ of carotid body tumours. ${ }^{2}$

\section{Vagal paraganglioma}

The vagal paraganglioma appears similar to the carotid body tumour with some exceptions. These masses displace both the external carotid artery (ECA) and internal carotid artery (ICA) anteromedially, separating these vessels from the internal jugular vein (IJV). In addition, extension into the suprahyoid carotid space is seen in approximately two-thirds of vagal paraganglio$\operatorname{mas}^{2}$ (Fig. 2).

\section{Glomus jugulare}

Enhancing mass in the jugular foramen with permeative-destructive pattern of bone destruction is the typical CT feature of glomus jugulare (Figs $3 a$ and $b$ ). The tumour spreads along the paths of least resistance and is initially directed superiorly owing to the intrinsic weakness of this part of the jugular fossa (Fig. 4). Subsequently, the hypotympanum, mesotympanum, and the sinus tympani are invaded. Ossicular chain destruction is common. Inferior spread of the tumour produces infiltration of the IJV and infratemporal fossa. ${ }^{1}$

\section{Glomus tympanicum}

The glomus tympanicum tumour manifests as a small discrete mass arising from the cochlear promontory and confined to the tympanic cavity (Figs $5 \mathrm{a}$ and $\mathrm{b}$ ). Ossicular destruction is not typical, although encase-
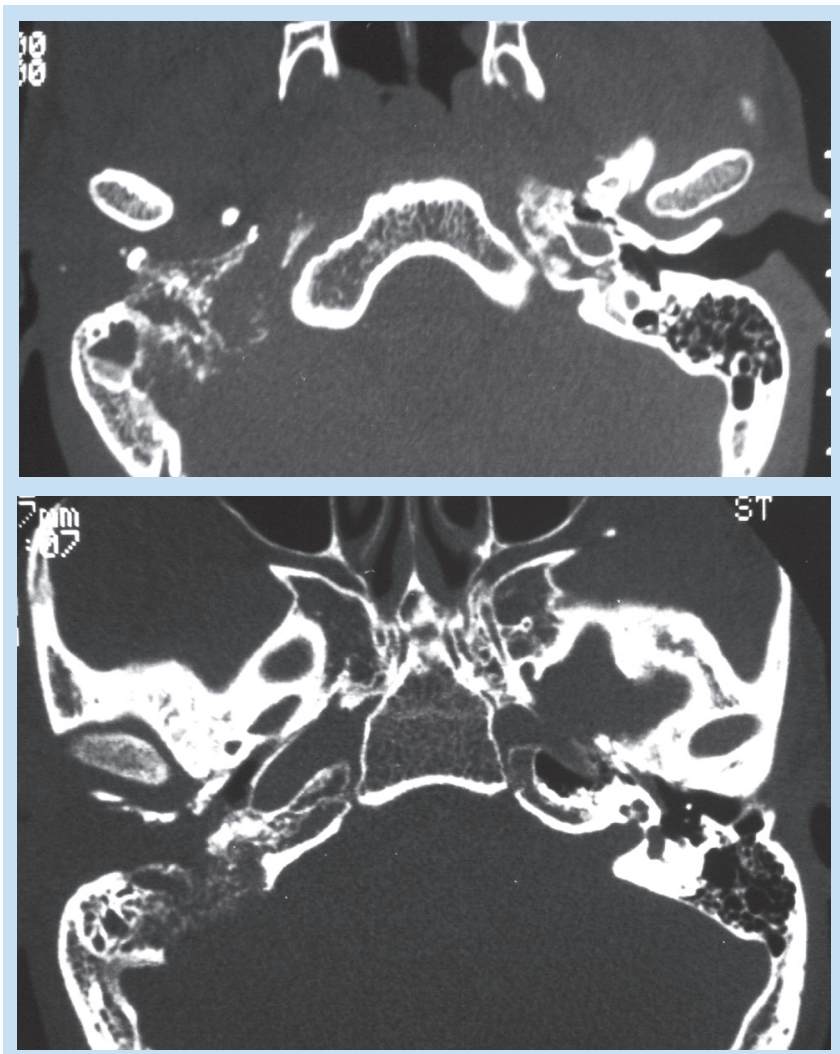

Fig. 3. Glomus jugulare. (a) Axial CT with bone windows shows permeative pattern of bone destruction at the base of the skull by the tumour. (b) Axial $\mathrm{CT}$ at a higher level shows bone destruction extending to the middle ear and mastoids.

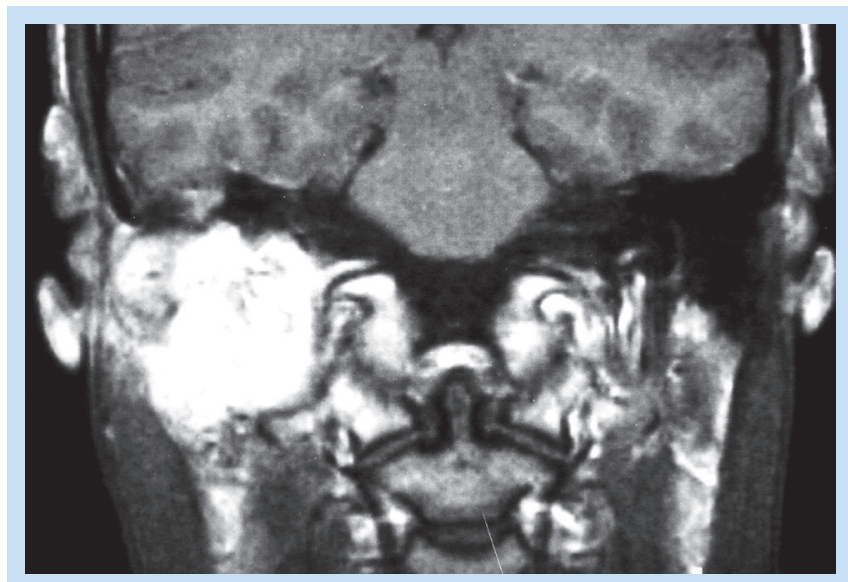

Fig. 4. Glomus jugulare. Postcontrast coronal MR sequence shows an enhancing mass with epicentre in the jugular foramen.

ment is frequent in larger lesions.

\section{MR imaging features}

Paragangliomas typically exhibit a low signal intensity with standard spin-echo on T1-weighted sequences and a high signal intensity with T2-weighted sequences. As with CT, a homogeneous and intense pattern of 

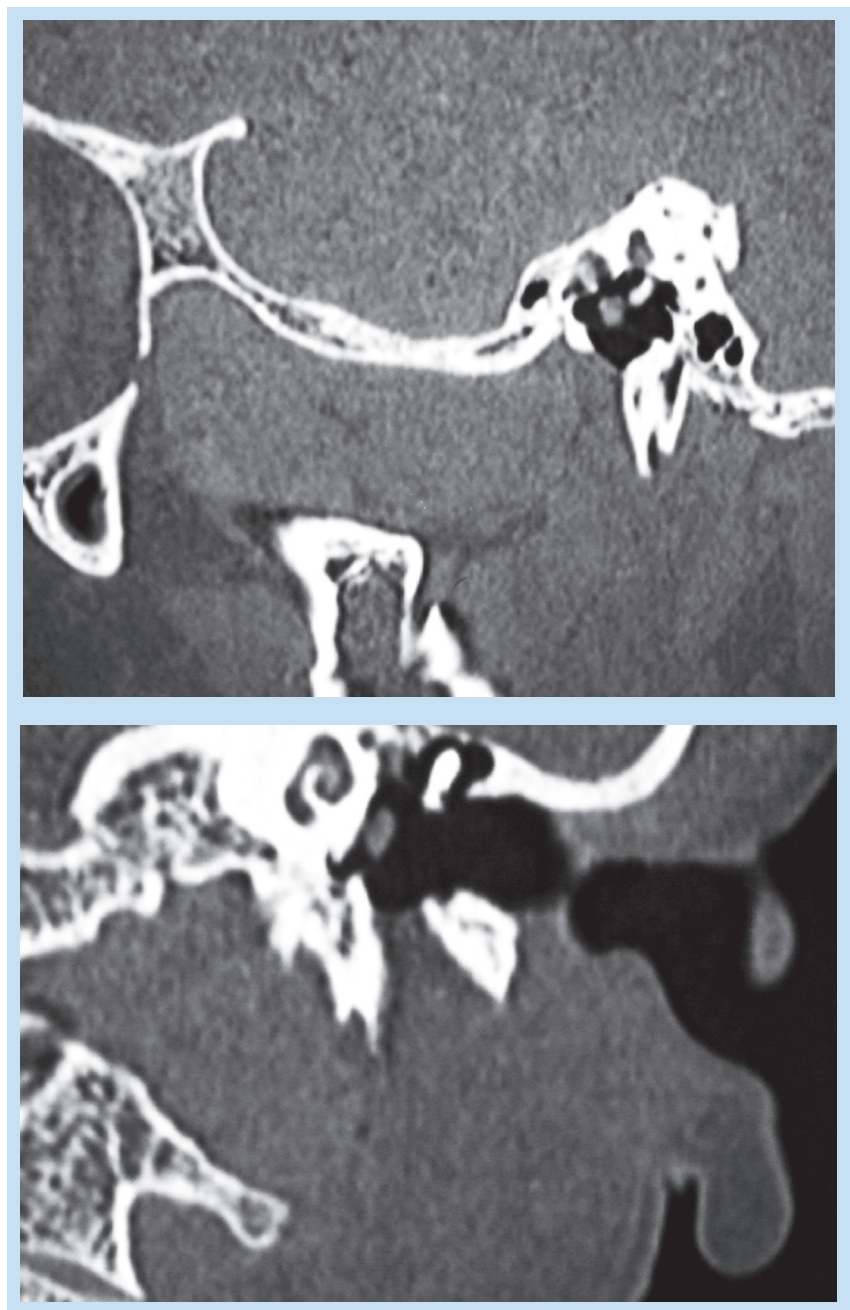

Fig. 5. Glomus tympanicum. (a) Sagittal and (b) coronal reconstructions of an HRCT through the temporal bone show a soft-tissue mass arising from the cochlear promontory.

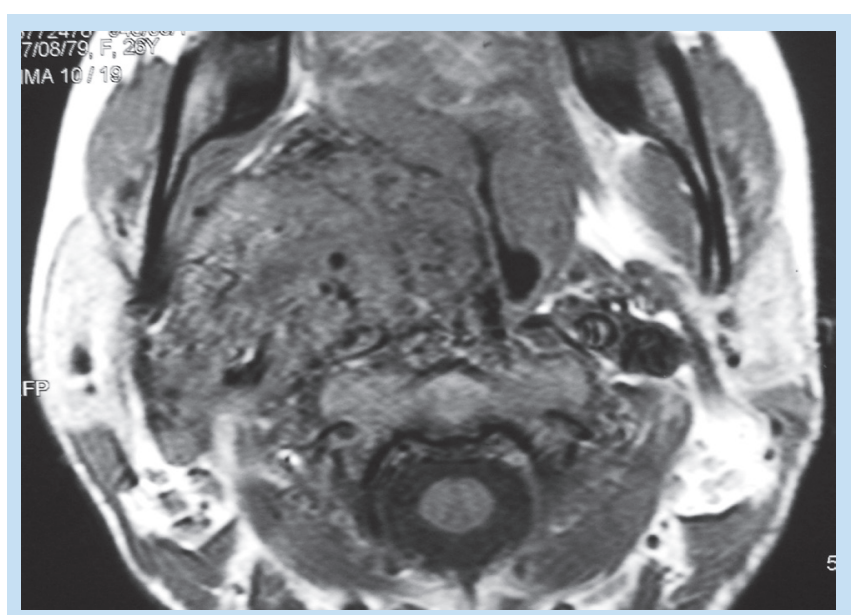

Fig. 6. Salt-and-pepper appearance of glomus tumour. Axial T1 sequence shows the typical salt-and-pepper appearance of a glomus tumour.

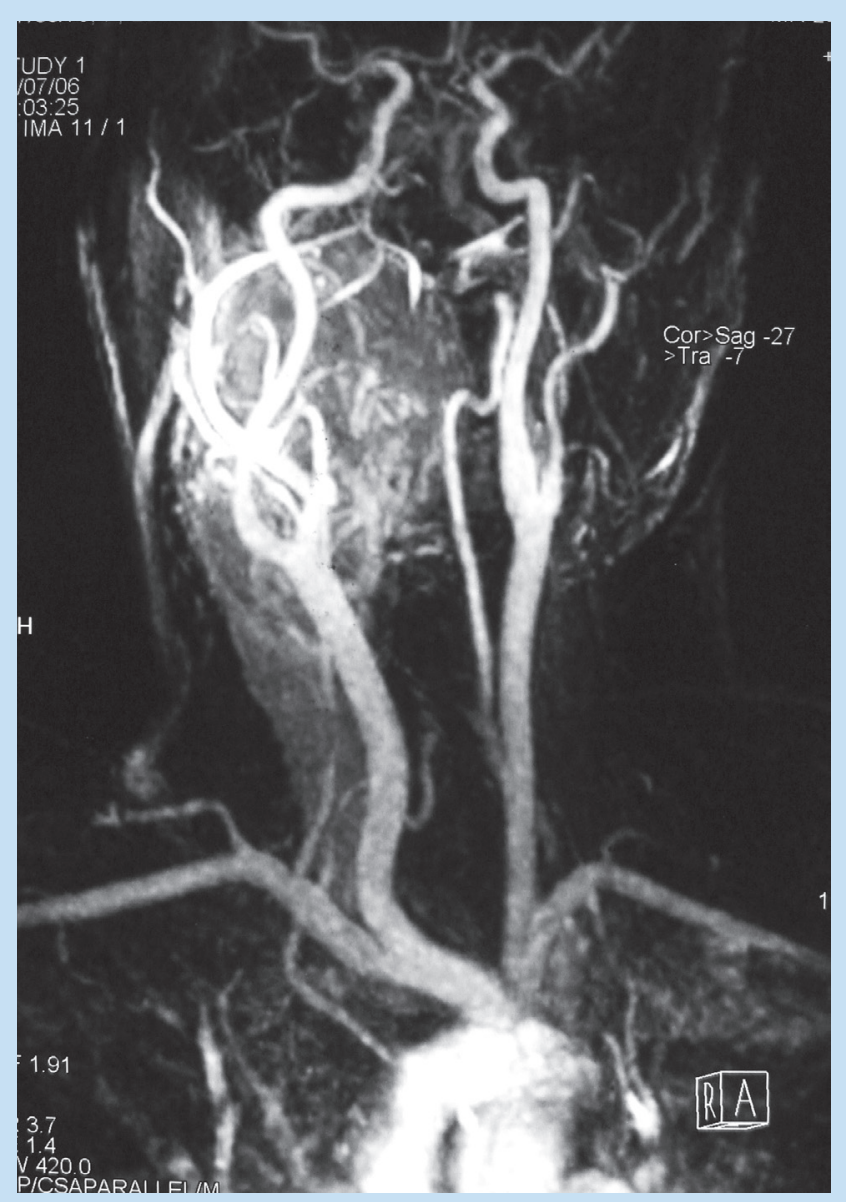

Fig. 7. Glomus vagale. Time of flight MRA studies show the typical hypervascularity of glomus tumours.

enhancement is noted following the intravenous administration of contrast material. The classic salt-and-pepper appearance in which the 'pepper' component represents the multiple areas of signal void interspersed with the 'salt' component seen as hyperintense foci (caused by slow flow or haemorrhage) is seen on both short TR and long TR images (Fig. 6). MR angiography may be useful in defining the flow-related enhancement of feeding vessels in lesions larger than $1.5 \mathrm{~cm}^{3}$ (Fig. 7). MR venography can help differentiate the glomus jugulare tumour from non-neoplastic vascular anomalies (e.g. a high-riding or dehiscent jugular bulb, jugular venous thrombosis, and asymmetric enlargement of the jugular foramen). ${ }^{2}$ 


\section{Angiographic appearance}

The typical angiographic appearance of a paraganglioma is that of a hypervascular mass with enlarged feeding arteries, intense tumour blush, and early draining veins (Fig. 8). Rarely, avascular paragangliomas may occur.

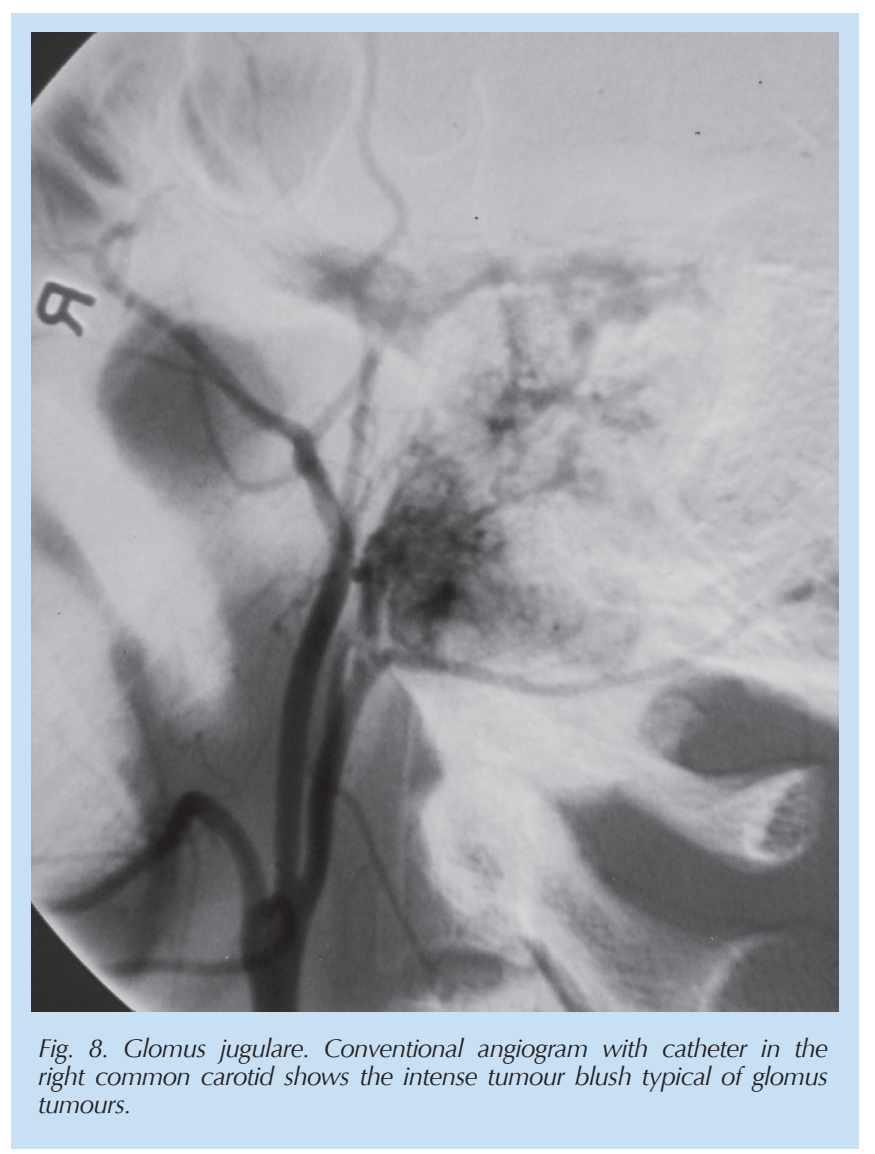

\section{Therapy}

Surgery is the treatment method of choice in most instances. Although most cervical paragangliomas are considered radioresistant, their skull base counterparts are known to be radiosensitive. In patients with unresectable tumours, residual tumour following surgery or tumour involvement that occludes the ICA, radiation therapy may serve as an excellent palliative modality.

Pre-operative embolisation has been acclaimed by many investigators as a useful adjunctive tool in the surgical management of paragangliomas. Shrinkage in tumour vascularity and size, with a consequent decrease in intra-operative blood loss, is the goal. It is believed that a tumour larger than $3 \mathrm{~cm}$ is ideally suited for embolisation. The success rate for pre-operative embolisation (as defined by a decrease in tumour size) is estimated at about $80 \%$. The recommended delay between embolisation and surgery should be at least 1-2 days to allow embolisation-related local oedema to decrease but no longer than 2 weeks to avoid recanalisation of the feeding vessels. ${ }^{4}$

\section{Conclusion}

In summary, paragangliomas are uncommon lesions of the head and neck region, constituting only $0.6 \%$ of all masses in this area. They are usually seen in four locations: the carotid body at the CCA bifurcation (carotid body tumour), the jugular foramen (glomus jugulare tumour), along the vagus nerve (vagal paraganglioma) and the middle ear (glomus tympanicum tumour). These hypervascular masses typically have a salt-andpepper appearance with prominent flow-voids on MR images. Intense enhancement is almost always noted following contrast material administration.

\footnotetext{
1. Rao AB, Koeller KK, Adair CF. Paragangliomas of the head and neck: radiologic-pathologic correlation. Radiographics 1999; 19: 1605-1632

2. Harnsberger R. Carotid space, neoplasm, benign tumors. In: Harnsberger R,Wiggins RH, Hudgins PA, et al. eds. Diagnostic Imaging. Head and Neck. Salt Lake City, Utah: AMIRYSYS, 2004; 8:16-23.

3. Som PM, Curtin HD. The parapharyngeal space. In: Som PM, Curtin HD, eds. Head and Neck Imaging. 3rd ed. Vol 2. St Louis, Mo: Mosby, 1996: 915-951.

4. Tikkakoski T, Luotonen J, Leinonen S, et al. Preoperative embolization in the management of neck paragangliomas. Laryngoscope 1997; 107: 821-826.
} 